Bull. Egypt. Soc. Physiol. Sci. Vol. (41) Issue (2), 203-219

\author{
Bull. of Egyp. Soc. Physiol. Sci. \\ (Official Journal of Egyptian Society for Physiological Sciences) \\ (pISSN: 1110-0842; eISSN: 2356-9514)
}

\title{
The Effect of Direct Acting Antiviral Therapy for Hepatitis C- Virus Infected Egyptian Patients on Changes in the Parameters of Liver Fibrosis Progression
}

\author{
Mohamed A. Elfiki ${ }^{1}$, Mahmoud Farid ${ }^{1}$, Ayman S. Soliman ${ }^{2 *}$, Bishoy A. Ibrahim ${ }^{3}$ \\ ${ }^{1}$ Internal Medicine Department, Faculty of Medicine, Beni-Suef University, Egypt. \\ ${ }^{2}$ Medical Physiology Department, Faculty of Medicine, Beni-Suef University, Egypt. \\ ${ }^{3}$ Gastroenterology and Hepatology Department, Faculty of Medicine, Beni-Suef University, Egypt.
}

Submit Date: May 20, 2020

Accept Date: June 28, 2020

Available online: Jan 10, 2021

Keywords

$\mathrm{HCV}$

DAA therapy

Liver fibrosis

progression

Liver stiffness

measurement

Fibroscan

\begin{abstract}
Hepatitis C Virus (HCV) is a major health care concern worldwide. Egypt shows the highest worldwide HCV prevalence. Fibrosis progression is common in $\mathrm{HCV}$ and it is the most important prognostic factor in chronic HCV patients. The aim of this study was to investigate changes in some parameters of liver fibrosis progression after successful $\mathrm{HCV}$ eradication by direct acting antiviral (DAA) therapy in Egyptian patients. The study included 100 chronic HCV patients. liver stiffness measurement (LSM) was obtained by Transient elastography (TE) or Fibroscan before starting DAA treatment, after the end of 12 weeks of treatment and after achieving sustained virologic response 12 (SVR12). Based on baseline LSM, patients were stratified into F2, F3 and F4 groups (METAVIR), F0-F1 patients were excluded. LSM and laboratory data after the end of treatment and after achieving SVR12 was compared with that baseline values in each fibrosis group. Following DAA treatment, all patients achieved SVR12. Mean baseline LSM dropped from 13.5 to $10.1(\mathrm{kPa})$ post SVR12; the maximum change occurred in F2 patients $84.3 \%$ versus $84.2 \%, 43.3 \%$ in F3, and F4 patients respectively $(\mathrm{p}<0.001)$. At baseline, 30 patients were in the F4 group; only 11 patients $(43.3 \%)$ regressed to noncirrhotic range $(<12.5 \mathrm{kPa})$, while 19 patients $(56.7 \%)$ were still cirrhotic despite achieving SVR12 $(\mathrm{p}<0.001)$. Patients showed significant improvement in platelets count and decreased ALT enzyme levels after achieving SVR12 $(p<0.001)$. So, successful eradication of $\mathrm{HCV}$ results in significant LSM improvement; the best improvement occurs in F2 and F3 patients.
\end{abstract}

Corresponding author: Ayman Saied Soliman, Medical Physiology Department, Faculty of Medicine, Beni-Suef University, Egypt, Email: aymensaied2470@gmail.com ; Tel.: +201002100757 \&+201223657085 


\section{INTRODUCTION}

Hepatitis C Virus (HCV) is a common health problem in Egypt. Unfortunately, most of patients are unaware by their illness and many cases are discovered lately after occurrence of substantial liver fibrosis [1].

The World Health Organization estimate that hepatitis $\mathrm{C}$ virus (HCV) infects 71 million people globally and causes approximately 399,000 deaths annually due to the complications of progressive liver fibrosis, including decompensated cirrhosis and HCC. By bloodborne transmission, there were 1.75 million new $\mathrm{HCV}$ infections worldwide in 2015 , that is 23.7 per 100,000 people [2].

Recently, direct acting antivirals (DAA) drugs with direct activity against $\mathrm{HCV}$, were introduced for treatment of $\mathrm{HCV}$ with incredibly favorable results regarding viral cure. However, the beneficial effect of antiviral treatment on modification of the natural history of chronic $\mathrm{HCV}$, reduction of fibrosis and decreasing the subsequent HCV-related complications and mortality remain controversial $[3,4]$.

The prevalence of HCV infection in Egypt is the highest in the world $[5,6]$. Although screening for $\mathrm{HCV}$ is recommended [7,8] and the nowadays available screening test has a $97 \%$ sensitivity and $100 \%$ specificity $[9,10]$, up to $60 \%$ of cases in Egypt are diagnosed with decompensated cirrhosis or HCC mainly due to the lack of a national screening program $[11,12]$.

Inflammation is a protecting physiological process of the liver against most infections caused by viruses and tissue damage. Though persistent, uncontrolled inflammation is the distinguishing feature of severe liver damage related to $\mathrm{HCV}$ infection, involving fibrosis, cirrhosis and HCC [13].

Liver fibrosis can be defined as the excessive accumulation of extracellular matrix proteins as a result of the interaction of a number of different cell types that include macrophages, myofibroblasts and epithelial cells, leading to excess of fibrillar collagens (predominantly collagens I and III). Fibrogenesis is a dynamic and complicated process, mediated through necroinflammation and activation of hepatic stellate cells (HSC) [14].

The mechanism of fibrosis associating $\mathrm{HCV}$ is a multifaceted process, although $\mathrm{HCV}$ does not infect hepatic stellate cells [13]. HCV infection induces numerous factors in host cells, such as non-coding RNAs and cytokines/chemokines, which directly or through exosomes activate quiescent hepatic stellate cells. On the other hand, macrophages exposure to $\mathrm{HCV}$ induce soluble mediator like cysteine-cysteine chemokine ligand 5 (CCL5), which also stimulates HSCs for activation [15].

One of the most significant characteristics of HSC, when they are in a quiescent state, is the presence of lipid droplets in the cytoplasm containing vitamin $\mathrm{A}$ as a retinylpalmitate. After liver injury is established, HSC lose their lipid droplets and undergo significant morphological and functional changes, developing a myofibroblast-like cell phenotype and beginning excessive production of collagen, mainly type I; this process is known as activation [14].

However, activated HSC yet differs from myofibroblasts and smooth muscle cells in their vitamin content, contractile activity, and their 
susceptibility to some cytokines, specially to transforming growth factor- $\beta 1$ (TGF- $\beta 1$ ) [16].

Hepatic fibrosis, in some cases, is reversible; it can be said that in this disease, the main characteristic is the disordered accumulation of extra cellular matrix (ECM) fibrillar type proteins mainly collagen that reduces the fenestrations between the sinusoidal cells in order to eliminate them and decrease liver function, with a loss of physiological architecture and nodule formation regeneration [14].

Additionally, sinusoidal cells increase the expression of pro-inflammatory molecules that include intercellular adhesion molecule -1 (ICAM1), vascular endothelial cell growth factor (VEGF) and adhesion molecules presenting a neoangiogenesis and contraction of scar tissue which leads to elevated tissue tension [17].

Accumulation of fibrillar proteins is the consequence of an imbalance between ECM synthesis and degradation plus to a low formation of Matrix metalloproteinases (MMPs), or their inhibitors overproduction, tissue inhibitors of metalloproteinase (TIMPs), or profibrogenic cytokine as TGF- $\beta$ [14].

A major determinant of fibrosis reversion in $\mathrm{HCV}$ patients is reversion of activated stellate cells to a more quiescent state. [14]. Three main mechanisms in the resolution of fibrosis have been found. The first one is HSC becoming apoptotic, the second one is HSC becoming senescent, and the third is HSC reversing their phenotype to an inactivated state [18].

Still, prolonged liver injury results in the expanded thickening of septae due to enhanced cross-linking by tissue transglutaminases and resistance to proteolysis by metalloproteinases restricting the complete regression of fibrosis [13]. Often $\mathrm{HCV}$ associated hepatocellular carcinoma (HCC) is seen with a background of liver fibrosis or cirrhosis [19].

In spite of the fact that liver biopsy remains the gold standard for hepatic fibrosis evaluation, it is invasive and painful, with uncommon but possibly life-threatening complications besides to some limitations of this procedure that include interobserver and sampling variability which may lead to under staging of fibrosis. Moreover, liver biopsy cannot be utilized for mass screening in a nation with a very high prevalence of HCV like Egypt [20].

Restrictions of the liver biopsy have propelled research for an accurate noninvasive test would be helpful to evaluate the stage of hepatic fibrosis, particularly for therapeutic and prognostic implications. [8,20]. Transient elastography (TE) or Fibroscan has developed as the novel, noninvasive method of reference. It is the foremost broadly utilized and approved method tha $t$ measures liver stiffness based on utilizing elastic shear waves emitted from the vibrator attached to the ultrasound transducer probe [21].

Pulse-echo ultrasound acquisitions follow the shear waves, and the speed of these waves, specifically related to tissue stiffness, is measured. The harder the tissue, the faster the shear wave propagates. However, it cannot be applied in stout persons and patients with ascites. Kilopascal unit is utilized in expression of the liver stiffness measurement (LSM) [22,23].

This study was conducted to investigate the changes in some parameters of liver fibrosis 
progression after successful HCV eradication by direct acting antiviral therapy in Egyptian patients.

\section{Subjects and Methods:}

\section{Subjects:}

The current study was conducted on 100 patients (64 males and 36 females) recruited from The Egyptian Liver Foundation, Beni-Suef. Their ages ranged from 18-70 years old. This work was done during the year 2019. The participants were previously diagnosed as chronic viral hepatitis $\mathrm{C}$ (HCV) patients on clinical and laboratory bases and they were candidates for direct acting antiviral agents. According to estimated METAVIR score based on baseline LSM, patients were classified into; 51 patients $(51 \%)$ were in F2, 19 patients $(19 \%)$ in $\mathrm{F} 3$, and 30 patients $(30 \%)$ in $\mathrm{F} 4$ fibrosis groups.

\section{Inclusion criteria:}

- Hepatitis C-infected patients with positive RNA - PCR.

- Patient Ages ranged from 18 to 70 years.

- Liver fibrosis stage ranged from F2 to F4.

- Patients with compensated liver disease.

- Treatment naïve patients.

\section{Exclusion criteria:}

- Patients ages less than 18 years old or more than 70 years old.

- Liver fibrosis stage F0 and F1.

- Patients with decompensated liver disease.

- Patients with Hepato-Cellular Carcinoma (HCC) and other patients not eligible for DAA therapy.

- Alcoholics and / or intravenous drug abusers.

\section{Ethical clearance:}

The study protocol was approved by the Research Ethical Committee of Faculty of Medicine, BeniSuef University (FWA \#: FWA00015574) at 9th of Dec 2018. A written informed consent was obtained from each patient enrolled in the study.

\section{Methods:}

All patients were subjected to liver stiffness measurement (LSM) was determined by using Transient elastography (TE) Fibroscan device (Fibroscan, Echosens, Paris, France), clinical and laboratory investigations which were done before starting DAA treatment, repeated after the end of treatment and then repeated after achieving SVR12.

\section{I- Thorough history taking:}

Including course of the disease and complications if found and drugs intake.

\section{I-Full clinical examination:}

Including general examination, chest, heart and abdominal examination.

\section{III- Laboratory investigations:}

\section{Sample collections:}

An overnight fasting (8-10 hours) venous blood sample was collected from each subject using a sterile plastic syringe. One portion of the collected blood was taken on Ethylene-diamine-tetra-acetic acid (EDTA) containing tube for CBC. The remainder of the blood was allowed to clot for 1020 minutes at room temperature then centrifuged for 20 minutes at the speed of 2000-3000 r.p.m to separate the serum. One portion of the serum was used immediately for the measurement of alanine transferase enzyme, total bilirubin, creatinine and albumin. The other portion was stored in epindorph tubes in a deep freeze at $-20^{\circ} \mathrm{C}$ till tested 
for hepatitis $\mathrm{C}$ virus RNA by polymerase chain reaction (PCR). Serum samples are stable for 3 months at $-20^{\circ} \mathrm{C}$.

\section{Biochemical assays:}

Complete blood count (CBC), serum alanine transferase enzyme $(\mathrm{U} / \mathrm{L})$, serum total bilirubin $(\mathrm{mg} / \mathrm{dL})$, serum albumin $(\mathrm{gm} / \mathrm{dL})$, serum creatinine (mg/dL) and hepatitis C virus RNA by polymerase chain reaction (PCR). All previously mentioned biochemical assays were done for all samples of the study groups. For all biochemical assays, the manufacturer instructions were followed.

\section{Abdominal ultrasonography:}

All patients underwent abdominal ultrasonography to assess the presence of liver cirrhosis, liver mass lesions, portal vein diameter, splenic size and the presence of ascites.

\section{Transient elastography (Fibroscan):}

Liver stiffness measurement (LSM) was determined by using Transient elastography (TE) Fibroscan device (Fibroscan, Echosens, Paris, France) by an expert Fibroscan operator, who was blinded about patient's data and the outcome of DAA treatment. Patient was positioned in supine position and the maximum abduction of his right arm. The probe tip was covered by gel and positioned at intercostal space in the right mid axillary line; right hepatic was used in measurement. The operator chose a large vesselsfree liver portion ( $\geq 6 \mathrm{~cm}$ in thickness) under guidance of time-motion ultrasound images.

After selection of the area for measurement, the probe button was pressed to begin the acquisition. Liver stiffness was measured in a tissue cylinder with width and length of 1 and $4 \mathrm{~cm}$ respectively, between 25 and $65 \mathrm{~mm}$ below the surface of skin.
The measurements with incorrect vibration shape or which do not have a correct follow up of the vibration propagation were automatically rejected by the software. Up to 10 valid measurements were done in every subject.

The ratio of the valid measurements to total acquisitions numbers was used as an indicator for the success rate. Reliable examination was considered if a success rate of $60 \%$, an interquartile range (variability in the validated measures) $<30 \%$ of the median elasticity and obtained Body Mass Index (BMI) less than 30 $\mathrm{kg} / \mathrm{m}^{2}$. The median liver stiffness value, expressed in kilopascals $(\mathrm{kPa})$, was considered to be representative of the elastic modulus of the liver [23].

Liver stiffness measurement (LSM) was used to estimate the METAVIR fibrosis stage as follows: F0-F1: 2.5-6.9 kPa; F2: 7.0-9.5 kPa; F3: 9.6-12.4 $\mathrm{kPa} ; \mathrm{F} 4: \geq 12.5 \mathrm{kPa}$. Where F0 denotes no fibrosis and F1 indicates portal fibrosis without septa. Significant fibrosis refers to a METAVIR score of F2 or greater whereas METAVIR F4 denotes cirrhosis [24].

\section{Direct acting antiviral (DAA) treatment}

DAA were used according to the functional state of the patient, including Sofosbuvir $400 \mathrm{mg}$ tablets in a dose of $400 \mathrm{mg}$ / day orally and Daclatasvir 60 $\mathrm{mg}$ tablets given once daily orally with or without Ribavirin $400 \mathrm{mg}$ tablets given twice daily orally for 12 weeks. Sustained virologic response 12 (SVR12) was defined as sustained loss of HCVRNA at 12 weeks after the end of treatment. 


\section{Statistical analysis}

Data were statistically described in terms of mean \pm standard deviation $( \pm S D)$, or frequencies (number of cases) and percentages when appropriate. For comparison of serial measurements within each patient the nonparametric Friedman test and Wilcoxon signed rank test were used. For comparing categorical data, Chi square $(\chi 2)$ test was performed. Exact test was used instead when the expected frequency is less than 5. $\mathbf{P}$ values less than 0.05 were considered statistically significant. All statistical calculations were done using computer program SPSS (Statistical Package for the Social Science; SPSS Inc., Chicago, IL, USA) release 15 for Microsoft Windows (2007).

\section{Results:}

A total of 100 (64 males; $64 \%$ and 36 females;36\%) Egyptian patients suffering of chronic HCV were included in this study. Table (1) demonstrates the baseline demographic and laboratory data of the study population.

According to estimated METAVIR score based on baseline LSM, the study population are classified into; 51 patients (51\%) were in F2, 19 patients (19\%) in $\mathrm{F} 3$, and 30 patients $(30 \%)$ in $\mathrm{F} 4$ fibrosis groups.

As illustrated in the Table (2) \& fig. 1, the achievement of SVR12 was associated with statistically significant reduction of mean serum alanine transaminase (ALT) levels from $38.13 \pm$ $23.4(\mathrm{U} / \mathrm{L})$ at baseline; before treatment to reach $19.08 \pm 9.6(\mathrm{U} / \mathrm{L})$ at the end of treatment period. More decline in mean serum alanine transaminase (ALT) levels had occurred 12 weeks after the end of treatment period to reach $15.43 \pm 6.2(\mathrm{U} / \mathrm{L})(\boldsymbol{p}$ $<\mathbf{0 . 0 0 1 )}$.

Additionally, Table (2) \& fig. 2, indicated that there was statistically significant elevation of mean serum bilirubin level from $0.62 \pm 0.3(\mathrm{mg} / \mathrm{dL})$ at baseline (before treatment) to reach $0.78 \pm 0.3$ $(\mathrm{mg} / \mathrm{dL})$ at the end of treatment period. While, the mean serum bilirubin level had declined 12 weeks after the end of treatment period to reach $0.67 \pm$ $0.2(\mathrm{mg} / \mathrm{dL})(\boldsymbol{p}<\mathbf{0 . 0 0 1})$.

Table (2) \& fig. 3, showed that there was statistically significant decrease in mean blood platelets level from $234.49 \pm 72.0\left(\mathrm{x} 10^{3} / \mathrm{dL}\right)$ at baseline (before treatment) to reach $195.98 \pm 61.4$ (x $\left.10^{3} / \mathrm{dL}\right)$ at the end of treatment period. While, the mean blood platelets levels had increased significantly 12 weeks after the end of treatment period to reach $247.09 \pm 68.6\left(\mathrm{x} 10^{3} / \mathrm{dL}\right)(p$ $<\mathbf{0 . 0 0 1 )}$.

There were fifteen patients (15\%) of our study population had pre-treatment thrombocytopenia, twelve of them (12\%) become within the normal range of platelet counts at SVR12 except only three patients (3\%) remain thrombocytopenic.

Moreover, Table (2) demonstrated that there was statistically significant elevation of mean total leukocyte count from $5.92 \pm 1.9\left(\mathrm{x} 10^{3} / \mathrm{dL}\right)$ at baseline (before treatment) to reach $6.68 \pm 2.1$ ( $\mathrm{x}$ $10^{3} / \mathrm{dL}$ ) at the end of treatment period. While, the mean total leukocyte count had declined 12 weeks after the end of treatment period to reach $6.48 \pm$ 1.5 (x 103/dL) $(\boldsymbol{p}<\mathbf{0 . 0 5})$.

Table (2) exhibited insignificant changes regarding hemoglobin levels as its levels were $13.67 \pm 1.6$, $13.19 \pm 1.4$ and $14.75 \pm 10.1(\mathrm{gm} / \mathrm{dL})$ at baseline, end of treatment period and 12 weeks after the end of treatment period respectively $(\boldsymbol{p}>\mathbf{0 . 0 5})$. 
In addition, there were insignificant decrease regarding serum albumin levels as its levels were $4.10 \pm 0.5,4.00 \pm 0.6$ and $3.97 \pm 0.4(\mathrm{gm} / \mathrm{dL})$ at baseline, end of treatment period and 12 weeks after the end of treatment period respectively $(p>$ 0.05).

Table (1): Baseline demographic and laboratory data of the included patients; $(N=100)$.

\begin{tabular}{|c|c|c|}
\hline Parameter & Mean \pm SD & (Max-Min) \\
\hline Age (years) & $50.5 \pm 8.5$ & $(70-18)$ \\
\hline BMI & $25.1 \pm 1.8$ & $(33.1-22.9)$ \\
\hline Hemoglobin; (g/dL) & $13.67 \pm 1.6$ & $(17.20-9.70)$ \\
\hline WBCs $\left(\times 10^{3} / \mathrm{dL}\right)$ & $5.92 \pm 1.9$ & $(14.00-2.40)$ \\
\hline Platelets $\left(\times 10^{3} / \mathbf{d L}\right)$ & $234.49 \pm 72.0$ & $(387.00-87.00)$ \\
\hline $\operatorname{ALT}(\mathbf{U} / \mathbf{L})$ & $38.13 \pm 23.4$ & $(124.00-13.00)$ \\
\hline Serum Bilirubin (mg/dL) & $0.62 \pm 0.3$ & $(2.30-0.20)$ \\
\hline Serum Albumin; (g/dL) & $4.1 \pm 0.5$ & $(5.40-2.30)$ \\
\hline Serum Creatinine; $(\mathrm{mg} / \mathrm{dL})$ & $0.78 \pm 0.2$ & $(1.30-0.50)$ \\
\hline
\end{tabular}

Table (2): Laboratory characteristics of the study population; $(\mathrm{N}=100)$ :

\begin{tabular}{|c|c|c|c|c|c|}
\hline \multirow[b]{2}{*}{ Time of Evaluation } & \multirow[b]{2}{*}{ Mean \pm SD } & \multicolumn{2}{|c|}{ 95\% CI for Mean ${ }^{1}$} & \multirow[b]{2}{*}{$\begin{array}{c}\text { Range } \\
\text { (Max-Min) }\end{array}$} & \multirow[b]{2}{*}{ p-value } \\
\hline & & $\begin{array}{l}\text { Lower } \\
\text { Bound }\end{array}$ & $\begin{array}{l}\text { Upper } \\
\text { Bound }\end{array}$ & & \\
\hline \multicolumn{6}{|l|}{ (a)- Hemoglobin; (gm/dL) } \\
\hline Baseline & $13.67 \pm 1.6$ & 13.36 & 13.98 & $7.50(17.20-9.70)$ & 0.169 \\
\hline End of Treatment & $13.19 \pm 1.4$ & 12.91 & 13.47 & $8.00(17.20-9.20)$ & \\
\hline $\begin{array}{c}12 \text { Weeks after ttt. } \\
\text { (b)- WBCs }\left(\times 10^{3} / \mathrm{dL}\right)\end{array}$ & $14.75 \pm 10.1$ & 12.74 & 16.77 & $105.60(14.30-8.70)$ & \\
\hline Baseline & $5.92 \pm 1.9$ & 5.52 & 6.31 & $11.60(14.00-2.40)$ & $0.013 *$ \\
\hline End of Treatment & $6.68 \pm 2.1$ & 6.27 & 7.08 & $12.10(14.80-2.70)$ & \\
\hline 12 Weeks after ttt. & $6.48 \pm 1.5$ & 6.18 & 6.78 & $9.40(12.90-3.50)$ & \\
\hline \multicolumn{6}{|l|}{ (c)- Platelets $\left(\times 10^{3} / \mathrm{dL}\right)$} \\
\hline $\begin{array}{l}\text { Baseline } \\
\text { End of Treatment } \\
12 \text { Weeks after ttt. } \\
\text { (d)- ALT; (U/L) }\end{array}$ & $\begin{array}{l}234.49 \pm 72.0 \\
195.98 \pm 61.4 \\
247.09 \pm 68.6\end{array}$ & $\begin{array}{l}220.20 \\
183.80 \\
233.47\end{array}$ & $\begin{array}{l}248.77 \\
208.15 \\
260.70\end{array}$ & $\begin{array}{l}300.00(387.00-87.00) \\
312.00(385.00-73.00) \\
294.00(382.00-88.00)\end{array}$ & $<0.001 *$ \\
\hline \multirow{2}{*}{$\begin{array}{l}\text { Baseline } \\
\text { End of Treatment } \\
12 \text { Weeks after ttt. }\end{array}$} & $\begin{array}{l}38.13 \pm 23.4 \\
19.08 \pm 9.6\end{array}$ & $\begin{array}{l}33.48 \\
17.16\end{array}$ & $\begin{array}{l}42.77 \\
20.99\end{array}$ & $\begin{array}{l}111.00(124.00-13.00) \\
60.00(67.00-7.00)\end{array}$ & $<0.001 *$ \\
\hline & $15.43 \pm 6.2$ & 14.19 & 16.66 & $27.00(34.00-7.00)$ & \\
\hline \multicolumn{6}{|c|}{ (e)- Serum Bilirubin (mg/dL) } \\
\hline $\begin{array}{l}\text { Baseline } \\
\text { End of Treatment }\end{array}$ & $\begin{array}{l}0.62 \pm 0.3 \\
0.78 \pm 0.3\end{array}$ & $\begin{array}{l}0.57 \\
0.71\end{array}$ & $\begin{array}{l}0.67 \\
0.84\end{array}$ & $\begin{array}{l}2.10(2.30-0.20) \\
2.60(3.10-0.50)\end{array}$ & $<0.001 *$ \\
\hline 12 Weeks after ttt. & $0.67 \pm 0.2$ & 0.63 & 0.71 & $1.50(2.00-0.50)$ & \\
\hline \multicolumn{6}{|c|}{ (f)- Serum Albumin; (gm/dL) } \\
\hline Baseline & $4.10 \pm 0.5$ & 3.78 & 5.41 & $4.70(5.40-2.30)$ & 0.274 \\
\hline End of Treatment & $4.00 \pm 0.6$ & 3.31 & 4.68 & $3.50(4.90-2.30)$ & \\
\hline 12 Weeks after ttt. & $3.97 \pm 0.4$ & 3.89 & 4.04 & $3.80(5.00-2.80)$ & \\
\hline
\end{tabular}

${ }^{I} \mathrm{CI}=$ Confidence Interval ${ }^{\dagger}$ Analysis done by Friedman test $* P$-value $<0.05$ is considered significant 


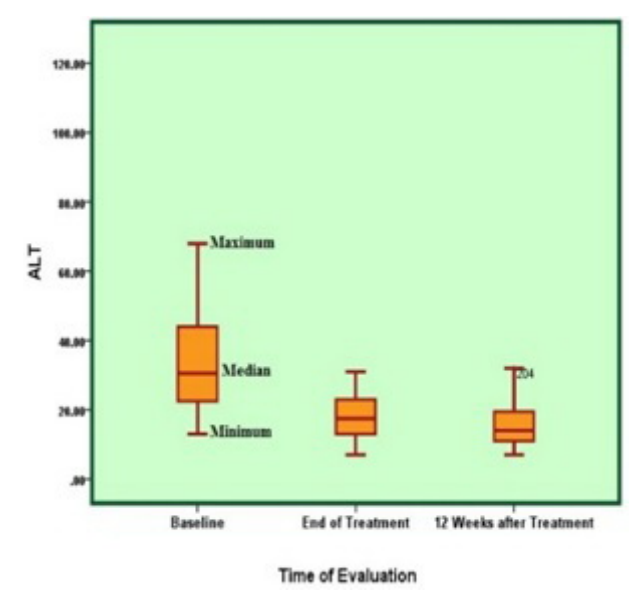

Figure 1: achievement of SVR-12 was associated with statistically significant reduction of mean serum ALT level from $38.13 \pm 23.4(\mathrm{U} / \mathrm{L})$ at baseline; before treatment to reach $19.08 \pm 9.6(\mathrm{U} / \mathrm{L})$ at the end of treatment period. More decline in mean serum ALT level had occurred 12 weeks after the end of treatment period to reach $15.43 \pm 6.2(\mathrm{U} / \mathrm{L})(\boldsymbol{p}<\mathbf{0 . 0 0 1})$.

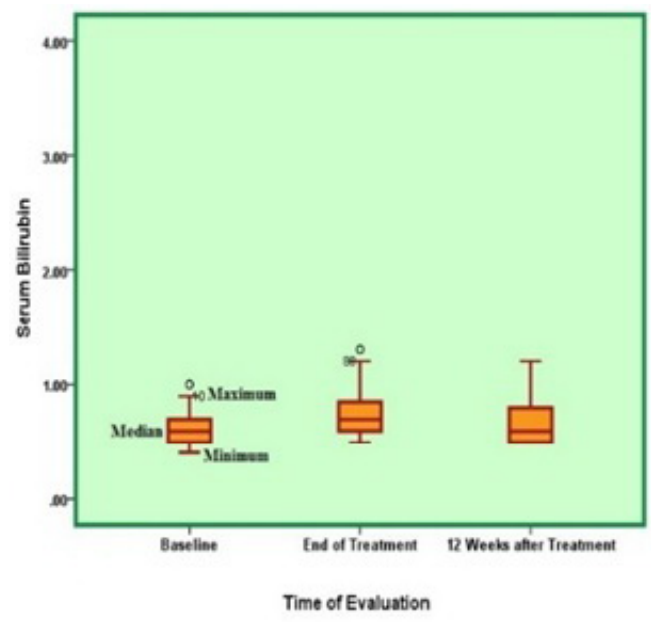

Figure 1: demonstrated that there was statistically significant elevation of mean serum bilirubin level from $0.62 \pm 0.3$ $(\mathrm{mg} / \mathrm{dL})$ at baseline (before treatment) to reach $0.78 \pm 0.3(\mathrm{mg} / \mathrm{dL})$ at the end of treatment period. While, it had declined 12 weeks after the end of treatment period to reach $0.67 \pm 0.2(\mathrm{mg} / \mathrm{dL})(\mathbf{p}<\mathbf{0 . 0 0 1})$.

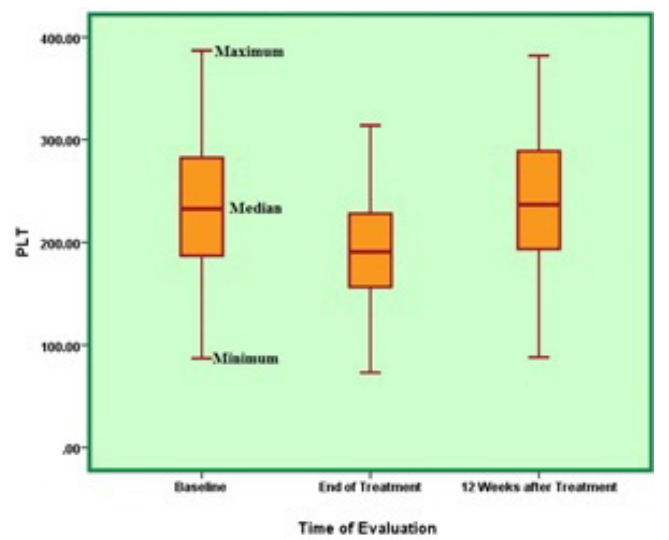

Figure 2: showed that there was statistically significant decrease in mean blood platelets level from $234.49 \pm 72.0$ (x $103 / \mathrm{dL})$ at baseline to reach $195.98 \pm 61.4$ (x 103/dL) at the end of treatment period. While, it had statistically significant increase 12 weeks after the end of treatment period to reach $247.09 \pm 68.6$ (x 103/dL) $(\mathbf{p}<\mathbf{0 . 0 0 1})$. 
Table (3) \& fig. 4, demonstrated a statistically significant improvement in liver stiffness measurement LSM $(\mathrm{kPa})$ determined by transient elastography with the use of a Fibroscan device. Evaluation of at the baseline level showed a mean value $13.58 \pm 9.7(\mathrm{kPa})$. At the end of treatment, the mean value was $11.41 \pm 10.6(\mathrm{kPa})$. And by the end of 12 weeks after treatment, further reduction in liver stiffness was detected as the mean value was $10.16 \pm 10.2(\mathrm{kPa})(\boldsymbol{p}<\mathbf{0 . 0 5})$.

Table (4) \& fig. 5, illustrated significant regression of liver fibrosis assessment of the studied population by METAVIR staging following antiviral therapy among patients with cirrhosis $(\boldsymbol{p}$ $<0.001)$.

According to estimated METAVIR score based on baseline LSM, 51 patients (51\%) were in F2, 19 patients $(19 \%)$ in $\mathrm{F} 3$, and 30 patients $(30 \%)$ in $\mathrm{F} 4$ fibrosis groups.
While at the end of antiviral treatment there was a significant regression of liver fibrosis assessment of the studied population by METAVIR staging as 42 patients $(42 \%)$ were in F0-F1, 19 patients $(19 \%)$ in F2,13 patients (13\%) in F3, and $26(26 \%)$ in F4 fibrosis groups $(\boldsymbol{p}<\mathbf{0 . 0 0 1})$.

Furthermore, significant regression of liver fibrosis assessment of the studied population by METAVIR staging occurred 12 weeks after the end of treatment period as 54 patients $(54 \%)$ were in $\mathrm{F} 0-\mathrm{F} 1,14$ patients $(14 \%)$ in $\mathrm{F} 2,13$ patients $(13 \%)$ in $\mathrm{F} 3$, and 19 patients (19\%) in $\mathrm{F} 4$ fibrosis groups $(\boldsymbol{p}<\mathbf{0 . 0 0 1})$.

Definitely, the best improvement in LSM occurred in F2 and F3 patients with mean percent of $84.3 \%$ and $-84.2 \%$ respectively versus $-43.3 \%$ in F4 patients $(\boldsymbol{p}<\mathbf{0 . 0 0 1})$.

Table 3: Liver stiffness measurement LSM (kPa) of the studied population.

\begin{tabular}{|c|c|c|c|c|c|}
\hline \multirow[b]{2}{*}{ Time of Evaluation } & \multirow[b]{2}{*}{ Mean \pm SD } & \multicolumn{2}{|c|}{ 95\% CI for Mean ${ }^{1}$} & \multirow[b]{2}{*}{$\begin{array}{c}\text { Range } \\
\text { (Max-Min) }\end{array}$} & \multirow[b]{2}{*}{$p$-value } \\
\hline & & $\begin{array}{l}\text { Lower } \\
\text { Bound }\end{array}$ & $\begin{array}{l}\text { Upper } \\
\text { Bound }\end{array}$ & & \\
\hline Baseline & $13.58 \pm 9.7$ & 11.65 & 15.51 & $56.80(63.90-7.10)$ & $0.049 *$ \\
\hline End of Treatment & $11.41 \pm 10.6$ & 9.30 & 13.51 & $72.20(75.00-2.80)$ & \\
\hline 12 Weeks after ttt. & $10.16 \pm 10.2$ & 8.13 & 12.19 & $70.20(73.00-2.80)$ & \\
\hline
\end{tabular}

$\uparrow$ Analysis done by Friedman Test

$* P$-value $<0.05$ is considered significant

Table (4): Comparison among number of patients classified according to METAVIR staging in baseline, end of treatment and 12 weeks after treatment $\$ ;(N=100)$ :

Time of Evaluation

\begin{tabular}{lcc}
\multicolumn{1}{c}{ METAVIR Staging } & Baseline & End of Treatment \\
F0-F1: (2.5-6.9) kPa & $0(0 . \%)$ & $42(42 . \%)$ \\
F2: (7.0-9.5) kPa & $51(51 . \%)$ & $19(19 . \%)$ \\
F3: (9.6-12.4) kPa & $19(19 . \%)$ & $13(13 . \%)$ \\
F4:( $\geq \mathbf{1 2 . 5}) \mathbf{~ k P a}$ & $30(30 . \%)$ & $26(26 . \%)$
\end{tabular}

$\neq$ Defined according to the METAVIR classification system

†Analysis done by Chi Square Test

$* P$-value $<0.05$ is considered significant

$\begin{array}{ll}12 \text { Weeks after } & \\ \text { Treatment } & \text { p-value } \\ 54(54 . \%) & <\mathbf{0 . 0 0 1}^{\dagger} \\ 14(14 . \%) & \\ 13(13 . \%) & \\ 19(19 . \%) & \end{array}$




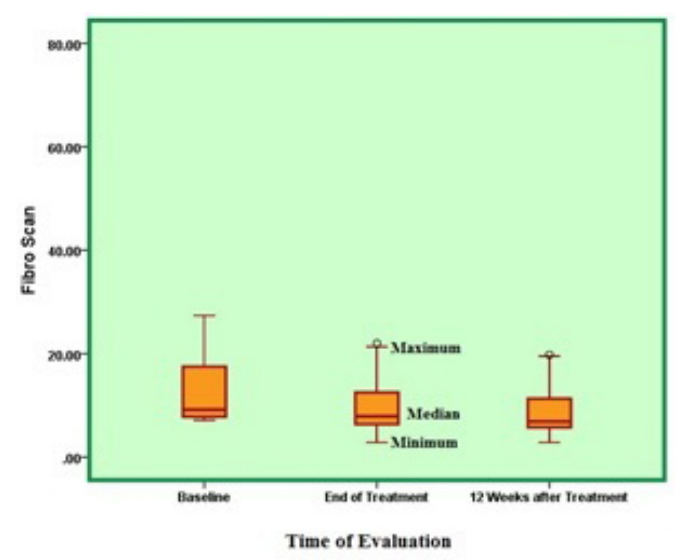

Figure 3: demonstrated a statistically significant improvement in liver stiffness measurement LSM (kPa) determined using a Fibroscan device. Baseline mean value of LSM was $13.58 \pm 9.7(\mathrm{kPa})$. At the end of treatment, the mean value was $11.41 \pm 10.6(\mathrm{kPa})$. And by the end of 12 weeks after treatment, further reduction in liver stiffness was detected as it reached $10.16 \pm 10.2(\mathrm{kPa})(\mathbf{p}<\mathbf{0 . 0 5})$.

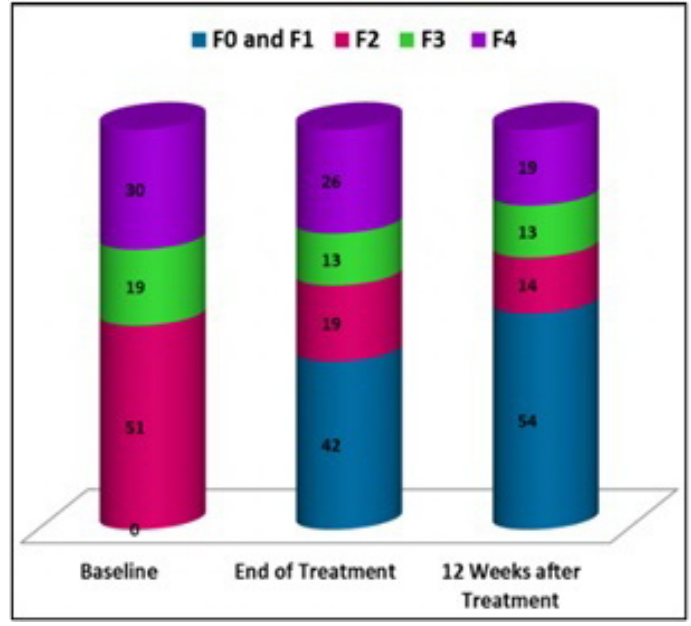

Figure 4: illustrated significant regression of liver fibrosis assessment of the studied population by METAVIR staging following antiviral therapy among patients with cirrhosis $(\boldsymbol{p}<\mathbf{0 . 0 0 1})$. The best improvement in LSM occurred in F2 and F3 patients with mean percent of $-84.3 \%$ and $-84.2 \%$ respectively versus $-43.3 \%$ in F4 patients $(\boldsymbol{p}<\mathbf{0 . 0 0 1})$.

\section{Discussion}

It has been proved that $\mathrm{HCV}$ eradication and achieving sustained virologic response12 SVR12 result in improved mortality and decreased risk in $\mathrm{HCV}$ related complications. Even though the mechanisms underlying such decrease in risk is yet to be fully determined, there is agreement that fibrosis regression plays a pivotal role [25].

SVR12 has been considered the goal of HCV treatment; however, liver fibrosis rather than serum viremia is the most important prognostic factor in chronic HCV patients [26].

Regression of liver fibrosis as a consequence of viral clearance is supported by the reduction of inflammatory mediators that leads to apoptosis of myofibroblasts and occurs by the inactivation of stellate cells. The downregulation of inflammation, along with microvascular remodeling, degradation of extracellular matrix and hepatocyte repopulation leads to the generation of new hepatic tissue [27]. Our study was conducted on 100 patients. It was limited to hepatitis C-infected Egyptian patients receiving DAA therapy and aimed to evaluate changes in some parameters of liver fibrosis progression after successful HCV eradication.

Because Liver Stiffness Measurement (LSM) is disease specific, only chronic HCV patients were 
included, while those with other liver diseases such as chronic HBV, HCC were excluded [28].

Our results demonstrated that achieving SVR12 was associated with statistically significant overall reduction of LSM from a mean value of $13.58 \pm 9.7$ $(\mathrm{kPa})$ in basal pre-treatment level to $10.16 \pm 10.2$ $(\mathrm{kPa})$ by the end of 12 weeks after treatment by a mean change of $-3.4 \pm 7.5 \quad(p<0.05)$ which is consistent with D'Ambrosio et al., [29] who showed a significant decrease in LSM with a median change from $10.40 \mathrm{kPa}$ to $7.60 \mathrm{kPa}$ $(\mathrm{p}<0.01)$ by a mean of $-2.8 \mathrm{kPa}$.

On the same line Tag-Adeen et al., [30] who had been shown a significant decrease in LSM by a mean of $-3.4 \mathrm{kPa}(\mathrm{P}<0.01)$, and Blach et al., [7] who showed a median decrease (pre-treatment to SVR48) from $8.0 \mathrm{kPa}$ (IQR: $5.0-11.9$ ) to $5.3 \mathrm{kPa}$ (IQR: 4.2-7.0), by a mean of $-3.7 \mathrm{kPa}(\mathrm{P}<0.01)$. However, our patients were treated with DAA, while it was interferon or interferon and DAA in the others.

Current study results showed that the best improvement in LSM occurred in F2 and F3 patients with mean percent of $-84.3 \%$ and $-84.2 \%$ respectively versus $-43.3 \%$ in $\mathrm{F} 4$ patients. In contrast with the study conducted by Sun and Kisseleva, [31] who showed more improvement in F4 patients with median percent changes $-44 \%$ in cirrhotic patients versus $-20 \%$ in non-cirrhotic patients. This discrepancy might have occurred because of the large number of F2 patients $(n=51)$ at the start of treatment in our study compared to F4 patients $(n=30)$. While in the study that was carried out by Sun and Kisseleva, [31] the number of pre-treatment F2 patient was 21 while F4 patients was 42 .
Despite the fact that it was accompanied by significant improvement in LSM and liver biochemistry, achieving SVR did not guarantee improvement of cirrhosis; only $43.3 \%$ of cirrhotic patients regressed to sub-cirrhotic range $(\mathrm{LSM}<12.5 \mathrm{kPa})$; relatively near results were obtained by many other previous studies.

Pritchett et al. [32] who compared changes in liver biopsies both pre- and post-treatment from 38 $\mathrm{HCV}$ patients, and they found cirrhosis regression in only $23(61 \%)$ patients.

On the same line with our results, Chekuri et al., [33] who perform their study on 84 chronic HCV patients and showed regression of cirrhotic to subcirrhotic range in only $39 \%$ of cirrhotic patients, also Sun and Kisseleva, [31] who conduct their study on $100 \mathrm{HCV}$ patients and they showed regression in only $40 \%$ of cirrhotic to a subcirrhotic range after SVR24 follow up.

So, most of cirrhotic (56.7\%) are still having risk for liver decompensation and HCC development despite achieving SVR12. This finding necessitates early treatment before occurrence of permanent liver damage to obtain the ultimate goal of $\mathrm{HCV}$ eradication.

Resolution of fibrosis following DAA therapy could be explained by three main mechanisms. The first one of these mechanisms is HSC becoming apoptotic, the second one is HSC becoming senescent and the third one is that HSC reversing their phenotype to an inactivated state [18].

One of the major goals in the fibrosis regression is to redirect the synthesis of inflammatory molecules to a restorative state. For that, healthy hepatocytes and non-parenchymal cells change the microenvironment from a pro-inflammatory 
ambience to resolution, in such a way that the presence of anti-inflammatory mediators increases. The liver macrophages become a restorative phenotype, increasing the expression of matrix metalloproteinases (MMPs) and growth factors leading to hepatocyte recovery [14].

In liver fibrosis, regression is enhanced, as the number of dendritic cells (DCs) and NK cells in the liver is increased. DCs favor matrix degradation through synthetic activation of matrix metalloproteinase -9 (MMP-9). A different cell type involved in regression are the NK cells, which induce the apoptosis of activated and senescent myofibroblasts. Then again, it is important to eliminate the excessive extracellular matrix: the MMPs play a very important role in this stage [18].

Some macrophages in the liver microenvironment release some anti-inflammatory mediators, as well as provide such fibrolytic mediators, like MMP-12 and MMP-13. Still, neutrophils and HSC can be expressed in other MMPs. But certain characteristics of advanced fibrosis adjudge some resistance to matrix degradation; these features are collagen cross-linking and deposition of elastin, among others [14].

Current study data indicated significant improvement in platelet counts (pre-treatment to SVR12) $(p<0.001)$. There were fifteen patients $(15 \%)$ of our study population had pre-treatment thrombocytopenia, twelve of them $(12 \%)$ become within the normal range of platelet counts at SVR12 except only three patients $(3 \%)$ remain thrombocytopenic.

In accordance with our results $H$ su et al. [34], who reported that the median platelet count was increased significantly from baseline 142 (99-190) (x $\left.10^{3} / \mathrm{dL}\right)$ to $151(109-195)\left(\mathrm{x} 10^{3} / \mathrm{dL}\right)$ at SVR 12 $(p<0.001)$. On the same line, Deterding et al. [35] and Pons et al. [36] both stated that platelets count increased significantly during treatment until 12 weeks after DAA therapy.

Common factors affecting platelet count in patients with $\mathrm{CHC}$ are liver fibrosis, hepatic necroinflammation [37], and thrombopoietin [38]. Karasu et al. [37] demonstrated that liver fibrosis stage was inversely associated with platelet count in patients with chronic hepatitis B and CHC.

Platelets interact with the hepatic sinusoidal endothelium while circulating in the injured liver and recruit effector cells and proteins [39]. This activity causes a self-perpetuating cycle of platelet and leukocyte accumulation, resulting in hepatocellular injury [40]. One study demonstrated that hepatic necro-inflammatory activity was correlated to a low platelet count in patients with CHC [41].

The current study results proved that the achievement of SVR12 was associated with statistically significant reduction of mean serum alanine transaminase (ALT) level as compared to its baseline levels $(p<0.001)$. Consistent with our results $H s u$ et al. [34], who demonstrated that the median serum (ALT) level was decreased from 66 (4 -102) at baseline level to $22(16-29)(\mathrm{U} / \mathrm{L})$ at SVR12 $(p<0.001)$. They explained this rapid decline in ALT levels due to improvement in necro-inflammation.

Similarly, Elsharkawy et al. [42], demonstrated a significant reduction in AST and ALT levels at week 4 in patients who received DAA therapy. They suggested that early reduction in AST and ALT levels mostly reflected reduction in 
necroinflammation rather than in fibrosis regression.

In our study, changes that have taken place in serum albumin levels were non-significant ( $p$ value $=0.274)$ comparing pre-treatment to SVR12, While Hézode et al., [43] stated significant improvement of serum albumin levels at SVR12 $(p<0.01)$ and described a marked increase in albumin levels at SVR12 in patients with a pretreatment albumin below 3.5g/dL. Such a difference could be attributed to the higher pretreatment albumin levels in our study as most of our patients $93 \%$ had a normal range $(\geq 3.5 \mathrm{~g} / \mathrm{dL})$ of serum albumin levels before beginning treatment.

The present study data demonstrated significant changes in mean serum bilirubin level as its level increased at the end of treatment then decreased again at SVR12 $(\mathrm{p}<0.001)$. These findings come in accordance with Deterding et al., [35] who reported that serum bilirubin level worsened during the first 12 treatment weeks $19 \pm 13 \mu \mathrm{mol} / \mathrm{L}$ vs. $23 \pm 18 \mu \mathrm{mol} / \mathrm{L} ; P=0.045$.

In addition, they reported that bilirubin levels returned to baseline levels during the follow-up period and importantly, even reached significantly lower levels 12 weeks after the end of treatment as compared to pre-treatment values in the subgroup of patients with abnormal bilirubin levels $35 \pm 12 \mu \mathrm{mol} / \mathrm{L}$ vs. $23 \pm 8 \mu \mathrm{mol} / \mathrm{L} ; P<0.001$.

Such alterations in serum bilirubin level could be explained on the basis that ribavirin-caused hemolysis as almost all patients received ribavirin as a part of their antiviral treatment regimen. Ribavirin causes hemolysis that leads to increased bilirubin levels during therapy particularly in patients with borderline hepatic function. This hemolytic effect of ribavirin stops after the end of treatment to completely disappear at SVR12 [35].

In our study changes in blood hemoglobin levels showed decline in mean blood hemoglobin levels at the end of treatment as compared to baseline levels. Meanwhile, it is increased at SVR12, but these changes did not reach significant value $(p-$ value $=0.169$ ). These findings come in agreement with Mai et al., [44] who reported that the mean blood hemoglobin is decreased significantly at the end of treatment $(\mathrm{p}<0.05)$.

These changes in blood hemoglobin level could be explained by the fact that ribavirin causes hemolysis leading to decreased blood hemoglobin level during therapy particularly in patients with borderline hepatic function. Then this hemolytic effect of ribavirin stops after end of treatment to disappear entirely at SVR12 [35].

The present study results showed significant increase in WBCs count comparing pre-treatment to end of treatment and SVR12 While [44] reported insignificant changes regarding WBCs count $(p>0.05)$. This difference could be attributed to the lower pre-treatment WBCs count in our study.

The main limitation of our study was the lack of histological evidence that confirms if LSM improvement could predominantly be related to the regression of fibrosis or resolution of necro inflammatory activity. Undoubtedly, invasiveness of liver biopsy with possibility of complications and disagreement of patients were main obstacles in doing liver biopsy. Also, US-based TE techniques are generally limited by the distance of liver from skin, high technical failure rates and confounding factors including obesity and hepatic inflammation [35]. 
Anyhow, many studies proved that measurements of liver stiffness obtained from Transient Elastography (Fibroscan) has a good correlation with METAVIR liver fibrosis score. Moreover, they reported that Fibroscan is a safe, rapid and non-invasive technique that might be useful for grading and staging of liver fibrosis with results that may be comparable to liver biopsy [23].

However, magnetic resonance elastography has a higher technical success and accuracy than these techniques, and it is now available in many leading institutions around the world, with growing clinical experiences and emerging new applications [45].

In conclusion, successful HCV eradication results in significant LSM improvement, the best improvement occurs directly after the end of treatment with more decrease in LSM after SVR 12. Also, the best improvement occurs in F2 patients. Unfortunately, the majority of cirrhotic patients are still having risk for liver decompensation and HCC development despite achieving SVR. Since our study found persistently elevated LSM in many patients, early diagnosis and treatment before fibrosis has been established is necessary to maximize the benefits of treatment. Nevertheless, patients with cirrhosis after SVR should be included in surveillance programs for cirrhosis complications and, especially, HCC risk. In this setting, we recommend that prospective studies are needed to determine the real risk of HCC occurrence and recurrence and the liver- and non-liver-related mortality after viral eradication.

\section{Conflicts of Interest}

None to declare

\section{References}

1- Adeel A, Peng Y, Samia A, Kenneth ES, Dawd S, Nasia S and BILAL H: Hepatitis C virologic response in hepatitis $\mathrm{B}$ and $\mathrm{C}$ coinfected persons treated with directly acting antiviral agents: Results from ERCHIVES. International Journal of Infectious Diseases; 92: 184-188, 2020.

2- Hsu S, Yang $S$ and Kao J: Risk of hepatocellular carcinoma development after hepatitis $\mathrm{C}$ virus eradicated by direct-acting antivirals: Fact or fiction? Journal of the Formosan Medical Association; 119: 3-11, 2020.

3- Martínez CJ, Bonis PS, Pons DM, Salcedo AMT, Mínguez RB and Genescà FJ: Transient elastography in DAA era. Relation between post-SVR LSM and histology. J Viral Hepat.; 27:453-455, 2020

4- Nahon P, Layese R, Bourcier V., Cagnot C, Marcellin P, Guyader D, et al.: Incidence of hepatocellular carcinoma after direct antiviral therapy for $\mathrm{HCV}$ in patients with cirrhosis included in surveillance programs. Gastroenterology, 155 (5):1436-1450, 2018.

5- Blach S, Zeuzem S, Manns M, et al.: Global prevalence and genotype distribution of hepatitis C virus infection in 2015: a modelling study. Lancet Gastroenterol Hepatol., 2(3):161-176, 2017.

6- Gomaa A, Allam N, Elsharkawy A, El Kassas M And Waked I: Hepatitis C infection in Egypt: prevalence, impact and management strategies. Hepat Med., 9:17-25, 2017. 
7- Razavi H, Robbins S, Zeuzem S, et al.: Hepatitis C virus prevalence and level of intervention required to achieve the WHO targets for elimination in the European Union by 2030: a modelling study. Lancet Gastroenterol Hepatol.; 2(5):325-336, 2017

8- Gamal S, Mahmoud S, Tarek $H$ and Dewolfe F: Mass screening for hepatitis B and $\mathrm{C}$ in Southern Upper Egypt. BMC Public Health, 19:1326-1333, 2019.

9- Smith BD, Morgan RL, Beckett GA, et al.: Centers for Disease Control and Prevention. Recommendations for the identification of chronic hepatitis $\mathrm{C}$ virus infection among persons born during 1945-1965. MMWR Recomm Rep., 61(43):886, 2012.

10-MOYER VA: U.S. Preventive Services Task Force. Screening for hepatitis $\mathrm{C}$ virus in adults. US Preventive Services Task Force Recommendations Statement. Ann Intern Med., 159 (5): 349-357, 2013.

11- Waked I, Doss W, El-Sayed MH, et al.: The current and future disease burden of chronic hepatitis $\mathrm{C}$ virus infection in Egypt. Arab $J$ Gastroenterol., 15(2): 45-52, 2014.

12- Estes C, Abdel-Kareem M, Abdel-Razek W, et al.: Economic burden of hepatitis $\mathrm{C}$ in Egypt: the future impact of highly effective therapies. Aliment Pharmacol Ther., 42(6): 696-706, 2015.

13-Mousumi K and Ratna BR: Mechanisms Underlying Hepatitis C Virus-Associated Hepatic Fibrosis. Cells; 8 (10): 1249, 2019.
14-Salas-Villalobosa TB, Lozano-Sepúlvedaa SA, Rincón-Sánchezb AR, Govea-Salasc M and Rivas-Estilla AM: Mechanisms involved in liver damage resolution after hepatitis $\mathrm{C}$ virus clearance. Med. Univ., 19:100-107, 2017.

15-Devhare PB, Sasaki R, Shrivastava S, Di Bisceglie AM, Ray $R$ and Ray RB: Exosome-mediated intercellular communication between hepatitis $\mathrm{C}$ virusinfected hepatocytes and hepatic stellate cells. J. Virol.; 91: 02216-02225, 2017.

16-Atzori L, Poli G and Perra A: Hepatic stellate cell: a star cell in the liver. Int $J$ Biochem Cell Biol.; 41:1639---42, 2009.

17-Pinzani M: Liver fibrosis in the post-HCV era. Semin Liver Dis.; 35:157---65, 2015.

18-Lee $Y$ and Friedman SL: Reversal, maintenance or progression: what happens to the liver after a virologic cure of hepatitis $C$ ? Antiviral Res.; 107:23-30, 2014.

19- Finkelmeier F, Dultz G, Peiffer KH, Kronenberger B, Krauss F, Zeuzem S, Sarrazin $\mathbf{C}$, Vermehren $\mathbf{J}$ and Waidmann O: Risk of de novo Hepatocellular Carcinoma after HCV Treatment with Direct-Acting Antivirals. Liver Cancer; 7(2):190-204, 2018.

20-Liu CH, Liu CJ, Hong CM, Su TH, Yang HC, Chen KM, Huang YP, Yeh YM, Tien HL, Liu YC, Kao JH, Chen DS and Chen PJ: A noninvasive diagnosis of hepatic fibrosis by BioFibroScore ${ }^{\circledR}$ in chronic hepatitis C patients. J Gastroenterol Hepatol.; 33 (1): 291-297, 2018.

21-Juan Fu, Biao Wu, Huazhi Wu, Feng Lin and Wei Deng: Accuracy of real-time 
shear wave elastography in staging hepatic fibrosis: a meta-analysis. BMC Med Imaging; 20: 16, 2020.

22-Sigrist RMS, Liau J, Kaffas AE, Chammas MC and Willmann JK: Ultrasound Elastography: Review of Techniques and Clinical Applications. Theranostics; 7(5):1303-1329, 2017.

23-Mona EH, Ahmed G, Tamer F and Mohamed H: Diagnostic value of Transient Elastography (Fibroscan) in the evaluation of liver fibrosis in chronic viral hepatitis $\mathrm{C}$ : Comparison to liver biopsy. The Egyptian Journal of Radiology and Nuclear Medicine; 48: 329-337, 2017.

24- Tsochatzis EA, Gurusamy KS, Ntaoula S, Cholongitas E, Davidson BR and Burroughs AK: Elastography for the diagnosis of severity of fibrosis in chronic liver disease: a meta-analysis of diagnostic accuracy. J Hepatol.; 54(4): 650-659, 2011

25- Gonzalez HC and Duarte-Rojo A.: Virologic cure of hepatitis $\mathrm{C}$ : impact on hepatic fibrosis and patient outcomes. Curr Gastroenterol Rep.;18(7): 32, 2016.

26-Cordero-Ruiz P, Carmona-Soria I, Rodríguez-Téllez M, Caunedo-Alvarez A, Quezada-Pacheco RH, Flores-Cucho A, Romero-Gómez $M$ and Vilches-Arenas Á: Long-term follow-up of patients with chronic hepatitis $\mathrm{C}$ treated with $\alpha$-interferon and ribavirin antiviral therapy: clinical and fibrosis impact of treatment response. Eur $J$ Gastroenterol Hepatol.; 29(7):792-799, 2017.

\section{7-Francesca RP, Francesca M, Cecilia} B, Maria AZ, Massimo S, Antonio G, Gian
LR, Maurizio P and Antonio G: Future of liver disease in the era of direct acting antivirals for the treatment of hepatitis C. World J Hepatol.; 9(7): 352-367, 2017.

28-Huang R, Rao H, Yang M, Gao Y, Wang J, Jin Q, Ma D And Wei L: Noninvasive Measurements Predict Liver Fibrosis Well in Hepatitis C Virus Patients After Direct-Acting Antiviral Therapy. Dig Dis Sci.; 65(5):14911500, 2020.

29- D'ambrosio R, Aghemo A, Rumi MG, et al.: A morphometric and immunohistochemical study to assess the benefit of a sustained virological response in hepatitis $\mathrm{C}$ virus patients with cirrhosis. Hepatology.; 56(2):532-543, 2012.

30- Tag-Adeen M, Sabra AM, Akazawa Y, Ohnita K and Nakao K: Impact of hepatitis C virus genotype-4 eradication following direct acting antivirals on liver stiffness measurement. Hepat Med.; Oct 6; 9:45-53, 2017.

31-Sun M and Kisseleva T: Reversibility of liver fibrosis. Clin Res Hepatol Gastroenterol; 39 Suppl 1: S60-S63, 2015.

32- Pritchett S, Cardenas A, Manning D, Curry $M$ and Afdhal NH: The optimal cut-off for predicting large esophageal varices using transient elastography is disease specific. $J$ Viral Hepat.;18(4): e 75-e80, 2011.

33-Chekuri S, Nickerson J, Bichoupan $\mathrm{K}$, et al.: Liver stiffness decreases rapidly in response to successful hepatitis $\mathrm{C}$ treatment and then plateaus. PLoS One, Jul 21;11(7): e 0159413, 2016 
34- Hsu W, Lai H, Su W, et al.: Rapid decline of noninvasive fibrosis index values in patients with hepatitis $\mathrm{C}$ receiving treatment with direct-acting antiviral agents. $B M C$ Gastroenterology; 19: 63, 2019.

35- Deterding K, Honer ZSC, Port K, Solbach P, Sollik L, Kirschner J, et al.: Improvement of liver function parameters in advanced HCVassociated liver cirrhosis by IFN-free antiviral therapies. Aliment Pharmacol Ther.;42:889901, 2015.

36- Pons M, Santos B, Simon-Talero $M$, Ventura-Cots M, Riveiro-Barciela M, Esteban R, et al.: Rapid liver and spleen stiffness improvement in compensated advanced chronic liver disease patients treated with oral antivirals. Ther Adv Gastroenterol.; 10:619-29, 2017.

37-Karasu Z, Tekin F, Ersoz G, Gunsar F, Batur Y, Ilter T, et al.: Liver fibrosis is associated with decreased peripheral platelet count in patients with chronic hepatitis B and C. Dig Dis Sci.; 52:1535-9, 2007.

38- Kaushansky K.: Thrombopoietin. $N$ Engl $J$ Med.; 339:746-54, 1998.

\section{9- Lalor PF, Herbert J, Bicknell R and Adams}

DH: Hepatic sinusoidal endothelium avidly binds platelets in an integrin-dependent manner, leading to platelet and endothelial activation and leukocyte recruitment. $\mathrm{Am} \mathrm{J}$
Physiol Gastrointest Liver Physiol.; 304: G469-78, 2013.

40-Chauhan A, Adams DH, Watson SP and Lalor PF: Platelets: no longer bystanders in liver disease. Hepatology.; 64:1774-84, 2016.

41-Streiff MB, Mehta $S$ and Thomas DL: Peripheral blood count abnormalities among patients with hepatitis $C$ in the United States. Hepatology.; 35: 947-52, 2002.

42- Elsharkawy A, Eletreby R, Fouad R, Soliman Z, Abdallah M, Negm M, et al.: Impact of different sofosbuvir based treatment regimens on the biochemical profile of chronic hepatitis C genotype 4 patients. Expert Rev Gastroenterol Hepatol.;11:773-8, 2017.

43-Hézode C, Castéra L, Roudot-Thoraval F, et al.: Liver stiffness diminishes with antiviral response in chronic hepatitis C. Aliment Pharmacol Ther.; 34(6): 656-663, 2011

44-Mai A, Ayman A, Eman A, et al.: High efficacy of generic and brand direct acting antivirals in treatment of chronic hepatitis C. International Journal of Infectious Diseases; 75: 109-114, 2018.

45- Maruoka D, Imazeki F, Arai M, Kanda T, Fujiwara $\mathrm{K}$ and Yokosuka O: Longitudinal changes of the laboratory data of chronic hepatitis $\mathrm{C}$ patients with sustained virological response on long-term follow-up. $J$ Viral Hepat.; 19(2): e97-104, 2012. 